\title{
Composición física y química de los suelos fluviovolcánicos de Armero Tolima, Colombia
}

\author{
Alfredo José Torres-Benítez ${ }^{1,2, *}$, Héctor Eduardo Esquivel ${ }^{2}$, Fernando Tinoco ${ }^{2}$ \\ ${ }^{1}$ Facultad de Ciencias Naturales y Matemáticas, Universidad de Ibagué, Ibagué, Colombia \\ ${ }^{2}$ Facultad de Ciencias, Universidad del Tolima, Ibagué, Colombia
}

\begin{abstract}
Resumen
Los cambios en la calidad de los suelos son determinados por su composición física y química y se relacionan directamente con el desarrollo, el crecimiento y los cambios en la dinámica de los procesos de sucesión vegetal. El objetivo de este estudio fue evaluar la composición física y química del suelo y su relación con la vegetación proveniente de procesos de sucesión ocurridos sobre los lodos fluviovolcánicos del municipio de Armero, Tolima, Colombia. Se establecieron 10 parcelas de 10 x 20 metros y en cada unidad de muestreo se dio apertura a tres calicatas para la obtención de una muestra compuesta. En cada muestra de suelo se determinaron los parámetros físicos y químicos y se estableció la relación entre las variables edáficas mediante un análisis multivariado de componentes principales. Las variables físicas, las densidades real y aparente, el porcentaje de porosidad y de humedad y las variables químicas: $\mathrm{pH}$, fósforo $(\mathrm{P})$, manganeso $(\mathrm{Mn})$, cinc $(\mathrm{Zn})$, calcio $(\mathrm{Ca})$, la saturación de bases: magnesio $(\mathrm{Mg})$, hierro $(\mathrm{Fe})$, cobre $(\mathrm{Cu})$, la relación de magnesio y potasio (relación $\mathrm{Mg} / \mathrm{K})$, la relación de calcio más magnesio con potasio $((\mathrm{Ca}+\mathrm{Mg}) / \mathrm{K})$, y la saturación y concentración de aluminio $(\mathrm{Al})$, presentaron alta variabilidad y valores sustentables para el desarrollo de la vegetación en la zona de Armero debido a los procesos ecológicos de sucesión. (C) 2017. Acad. Colomb. Cienc. Ex. Fis. Nat.
\end{abstract}

Palabras clave: Sucesión; Fisicoquímica; Parcelas; Parámetros físicos y químicos.

Physical and chemical composition of fluviovolcanic soils in Armero, Tolima, Colombia

\begin{abstract}
Changes in soil quality are determined by their physical and chemical composition and are directly related to the development, growth and changes in the dynamics of plant succession processes. The objective of this study was to evaluate the physical and chemical composition of the soil and its relationship with vegetation from successional processes developed on fluviovolcanic sludge in the municipality of Armero, Tolima, Colombia. Ten plots of 10x20 $\mathrm{m}$ were established and three trial pits were opened in each unit to obtain a composite sample. We determined physical and chemical parameters in each soil sample and we established the relationship between the edaphic variables through a multivariate analysis of principal components. Physical variables, actual and apparent density, porosity and humidity percentages and chemical variables: $\mathrm{pH}, \mathrm{P}, \mathrm{Mn}, \mathrm{Zn}, \mathrm{Ca}$, base saturation: $\mathrm{Mg}, \mathrm{Fe}, \mathrm{Cu}, \mathrm{Mg} / \mathrm{K}$ ratio, $(\mathrm{Ca}+\mathrm{Mg}) / \mathrm{K}$ ratio, and $\mathrm{Al}$ saturation and concentration presented high variability and sustainable values for the development of vegetation in Armero as a result of sequential ecological processes. C 2017. Acad. Colomb. Cienc. Ex. Fis. Nat.
\end{abstract}

Key words: Succession; Physical chemistry; Plots; Physical and chemical parameters.

\section{Introducción}

El suelo es un cuerpo natural, puede considerarse vivo, y está sujeto a la acción de los factores formadores (clima, organismos, material parental, tiempo y relieve); por lo que nace, crece, se reproduce y muere; en escalas temporales mayores a la humana; además, es también un recurso no renovable (Loaiza, 2011). Los procesos geodinámicos vulcanológicos son mecanismos naturales que renuevan la estructura de los suelos, su aporte de nutrientes es significativo y permiten, de ésta forma, a los grupos de especies vegetales; desarrollarse y adaptarse a condiciones edáficas específicas. Sin embargo, varios estudios (Cantillo, Lozada, Pinzón, 2009; Torres, et al., 2012) nos muestran como la composición y las características físicas y químicas de los suelos diseñan un patrón vegetal que predominará sobre otras especies, observándose cómo la sustentabilidad de un cultivo o un bosque de especies forestales, es inherente al estado de fertilidad del suelo que suministra los requerimientos

\footnotetext{
*Correspondencia:

Alfredo José Torres-Benítez, alfredo.torres@unibague.edu.co

Recibido: 22 de diciembre de 2016

Aceptado: 14 de marzo de 2017
} 
nutricionales de la planta. El conocimiento de los procesos de sucesión y edáficos en áreas afectadas por erupciones volcánicas en Colombia no se ha desarrollado suficientemente; por lo que, consideramos necesario fortalecer la investigación de éstos procesos de la dinámica ecológica.

Con el fin de caracterizar los sedimentos depositados por la avalancha en las tierras de Armero, Frye (1986) reportó en la caracterización física que el espesor del material varía entre 0,6 y $1,3 \mathrm{~m}$., la textura de los lodos es franco arenosa gravillosa con un contenido moderado de limo $(19,6 \%)$ y bajo de arcillas (12,5\%), lo que le confería una baja capacidad de absorción de agua y de nutrientes. En el análisis químico, reportó un $\mathrm{pH}$ de 3,9, una presencia importante de sales solubles con predominancia de sulfatos de $\mathrm{Ca}$ y $\mathrm{Mg}$, una reducida capacidad de intercambio catiónico y con respecto a características de fertilidad, reporta una buena capacidad de suministro de nutrientes, con excepción del $\mathrm{N}$ y el $\mathrm{P}$.

Cháves, Guevara (1987) realizaron la mapificación de los lodos con base en las fotografías aéreas tomadas y ampliadas por el IGAC. en 1985 y 1986, lo cual permitió delimitar los lodos en unidades cartográficas a nivel de consociación, procediendo luego a la selección de los sitios para la descripción, muestreo y clasificación de los perfiles que fueron confirmados mediante análisis de laboratorio.

Malagón (1989) caracterizó a los lodos de Armero de la siguiente forma: Con respecto a sus características físicas reportó un color pardo grisáceo oscuro los primeros 30 $\mathrm{cm}$.; una textura conformada por arena 70,6\%, limo 19,2\% y arcilla $10,2 \%$; fertilidad baja debida a la alta acidez, bajo contenido de material orgánico y de $\mathrm{N}$, valores bajos de cambio y salinidad ligera. En cuanto a las propiedades químicas, reportó un $\mathrm{pH}$ de 4,4 a 4,5 y una capacidad de intercambio catiónico baja de 9,1 m.e. $100 \mathrm{~g}^{-1}$.

En el primer estudio florístico de los lodos fluviovolcánicos, en la zona de Armero, Esquivel, Leguizamón (1989) citaron la descripción de las consociaciones y tierras de Ripio según el IGAC. En él se muestra la composición química del suelo y las principales especies desarrolladas en cada una de ellas. Las series de los lodos designados en éste estudio y que sirvieron como base para establecer las diferencias florísticas entre cada una de las consociaciones fueron: Serie Armero, Serie San Lorenzo, Serie El Puente, Serie La Playa, Serie Lavapatas, Serie Santuario, Serie La Estación, Serie Cofa y tierras de Ripio.

Castillo, Salinas (1992) en un estudio del análisis de los lodos de Armero y su vegetación en tres partes alrededor del río Lagunilla, reportaron en la parte alta una vegetación muy baja que se correlaciona con las características que presentaba el suelo: C.I.C. bajo, porcentaje de acidez alto y una alta concentración de Fe. En la parte media reportaron deficiencia de $\mathrm{K}$ consecuencia de la lixiviación y una alta concentración de Fe que causaba un suelo ligeramente ácido. En la parte baja reportaron un equilibrio entre elementos mayores como N, P y K, favoreciendo el desarrollo de cultivos.
En el segundo estudio de la sucesión vegetal de los lodos fluviovolcánicos de Armero, Esquivel, Ramírez (1994) reportaron la relación íntima entre el proceso de sucesión vegetal y los cambios de las características del suelo, como un incremento en la textura por la arcilla en las consociaciones con sucesión más avanzada, aumento del $\mathrm{pH}$ o disminución de la acidez con el incremento de la vegetación, disminución de la salinidad, disminución de Al, $\mathrm{Zn}, \mathrm{Fe}, \mathrm{Cu}$ y Mn, incremento de materia orgánica por el aumento de la vegetación, aumento del $\mathrm{K}$ a medida que avanzó el proceso sucesional, incremento amplio de $\mathrm{P}$ en las asociaciones constituidas por especies de gramíneas, tendencia a disminuir la presencia de $\mathrm{S}$, pero se mantuvo alto en el sector dominado por Cyperus ligularis (Mariscus ligularis), y tendencia a la disminución de $\mathrm{Mg}$. Esto es evidencia de que la composición física y química del suelo interviene en la continuidad del proceso sucesional.

En el tercer estudio del estado de sucesión vegetal de los lodos de Armero, Esquivel, Frye, Ramírez (1999), reportaron niveles de $\mathrm{pH}$ entre 3,6 a 6,3 favorables para el desarrollo de la vegetación, tendencia a niveles normales de salinidad, excepto en sectores dominados por Cyperus ligularis (Mariscus ligularis), incremento de $\mathrm{Al}$ y tendencia a la variación en la concentración de $\mathrm{S}$ activo entre 25 ppm a $625 \mathrm{ppm}$. Los objetivos del presente estudio fueron evaluar la composición física y química de los suelos en la zona de Armero (Tolima-Colombia), analizar su variación en el tiempo y su relación con la vegetación proveniente de procesos de sucesión vegetal.

\section{Materiales y métodos}

La zona de estudio se encuentra en el área de influencia de los lodos fluviovolcánicos en la margen izquierda del río Lagunilla en el municipio de Armero-Guayabal (TolimaColombia), inicialmente abarca una extensión de 3500 hectáreas que inician en el Piedemonte de la Cordillera Central a 400 metros de altura, extendiéndose $16 \mathrm{~km}$ hasta la orilla del río Magdalena a $280 \mathrm{~m}$ de altura y de sur a norte abarca $6 \mathrm{~km}$ desde el río Lagunilla hasta el río Sabandija ( $4^{\circ}$ $58^{\prime} \mathrm{N}$ y $\left.74^{\circ} 54^{\prime} \mathrm{O}\right)$. En la actualidad, la zona de estudio está notablemente reducida, debido a que en la mayor parte del área afectada se cultiva arroz (Oriza sativa), sorgo (Sorghum bicolor) y algodón (Gossypium herbaceum). La zona tiene una temperatura promedio de $27,8^{\circ} \mathrm{C}$, una precipitación promedio anual entre 1100 y $1960 \mathrm{~mm}$, la humedad relativa fluctúa entre $55 \%$ y el $83 \%$ y los valores de evaporación llegan a 1712.8 anual (Esquivel, et al., 1999). La región corresponde a la formación de bosque seco tropical según la clasificación de Holdridge (1979).

Caracterización de las parcelas de muestreo: las unidades de muestreo correspondieron a 10 parcelas de 10x20 metros, establecidas en los estudios realizados por Esquivel, Leguizamón (1989), Esquivel, Ramírez (1994) y Esquivel, et al., (1999) para la caracterización de la vegetación sucesional y el análisis edafológico de los lodos (Tabla 1). 
Tabla 1. Ubicación de las parcelas establecidas en los lodos fluviovolcánicos de Armero (Tolima-Colombia)

\begin{tabular}{cccc}
\hline \multirow{2}{*}{ Parcelas } & \multicolumn{2}{c}{ Coordenadas } & $\begin{array}{c}\text { Altura } \\
\text { (msnm) }\end{array}$ \\
\cline { 2 - 3 } & Norte & Oeste & 409 \\
\hline 1 & $4^{\circ} 57^{\prime} 49,5^{\prime \prime}$ & $7^{\circ} 55^{\prime} 25,7^{\prime \prime}$ & 410 \\
\hline 2 & $4^{\circ} 57^{\prime} 54,5^{\prime \prime}$ & $74^{\circ} 55^{\prime} 0,99^{\prime \prime}$ & 400 \\
\hline 3 & $4^{\circ} 57^{\prime} 48,5^{\prime \prime}$ & $74^{\circ} 55^{\prime} 30,6^{\prime \prime}$ & 410 \\
\hline 4 & $4^{\circ} 57^{\prime} 42,5^{\prime \prime}$ & $74^{\circ} 54^{\prime} 45,7^{\prime \prime}$ & 349 \\
\hline 5 & $4^{\circ} 57^{\prime} 52,8^{\prime \prime}$ & $74^{\circ} 53^{\prime} 56,4^{\prime \prime}$ & 385 \\
\hline 6 & $4^{\circ} 58^{\prime} 0,3^{\prime \prime}$ & $74^{\circ} 54^{\prime} 15,6^{\prime \prime}$ & 340 \\
\hline 7 & $4^{\circ} 59^{\prime} 55,2^{\prime \prime}$ & $74^{\circ} 54^{\prime} 28,5^{\prime \prime}$ & 380 \\
\hline 8 & $4^{\circ} 57^{\prime} 44,5^{\prime \prime}$ & $74^{\circ} 54^{\prime} 29,7^{\prime \prime}$ & 410 \\
\hline 9 & $4^{\circ} 57^{\prime} 43^{\prime \prime}$ & $74^{\circ} 53^{\prime} 57,1^{\prime \prime}$ & 335 \\
\hline 10 & $4^{\circ} 57^{\prime} 18,7^{\prime \prime}$ & $74^{\circ} 54^{\prime} 28,6^{\prime \prime}$ & \\
\hline
\end{tabular}

Fase de campo: se procedió a la apertura de tres calicatas por parcela para obtener tres submuestras que conformaron una muestra compuesta en cada una de las parcelas (ICA, 1992). Cada una de las calicatas contó con las siguientes especificaciones: $50 \mathrm{~cm}$ de ancho, $50 \mathrm{~cm}$ de largo y $70 \mathrm{~cm}$ de profundidad, elaborando una al extremo izquierdo, una en el centro y otra en el extremo derecho de la parcela, con el fin de tener una muestra representativa del suelo correspondiente al horizonte A que se identifica como el más sensible a las variaciones de la calidad del suelo (Arbelo, Guerra, Mora, Laguna, 2002).

Las 10 muestras de suelos se guardaron en bolsas estériles (Cuestas, 2005; Alfaro, Alvarado, Chaverri, 2001; FAO, 1990) previamente marcadas con el nombre del sitio y se trasladaron a los laboratorios de Análisis de Suelos de la Universidad del Tolima.

Fase de laboratorio: con cada una de las muestras se determinaron los parámetros físicos de textura, porosidad, humedad, densidad aparente y densidad real, consistencia, color y estructura, así como los parámetros químicos de $\mathrm{pH}$, materia orgánica, capacidad de intercambio catiónico, elementos mayores ( $\mathrm{P}, \mathrm{Ca}, \mathrm{Mg}, \mathrm{K}$,), elementos menores $(\mathrm{Na}, \mathrm{Fe}, \mathrm{Cu}, \mathrm{Zn}, \mathrm{Mn}, \mathrm{B}, \mathrm{S}, \mathrm{Al})$, saturación de aluminio, saturación de bases, relación $\mathrm{Ca} / \mathrm{Mg}$, relación $(\mathrm{Ca}+\mathrm{Mg}) / \mathrm{K}$ y relación $\mathrm{Mg} / \mathrm{K}$.

Análisis de los datos: se analizaron las características de los suelos de cada una de las parcelas mediante un Análisis de Componentes Principales (ACP) generado por el programa Infostat, para establecer relaciones entre las variables físicas y químicas. Se analizó la variación química del suelo a través del tiempo mediante un análisis descriptivo y su efecto sobre el proceso de sucesión vegetal.

\section{Resultados}

Relación entre variables físicas: los dos primeros factores del análisis de componentes principales (PCA) explicaron la variabilidad del 78\% (Figura 1). Las parcelas 3 y 5 aportaron un porcentaje de variación del $50 \%$ al factor 1 , mientras que las parcelas 8, 7, 4 y 2 aportaron un porcentaje de variación del $28 \%$ al factor 2 . Las variables densidad real (Dr), porcentaje de porosidad (\% P), densidad aparente (Da) y porcentaje de humedad $(\% \mathrm{H})$ fueron robustas y aportaron información para el análisis, sin embargo, no se presentaron agrupamientos significativos entre las variables.

Se encontró que la variable Da estaba influenciada por las parcelas 8, 7, 4 y 2, agrupando la mayor similaridad, y correlacionándose negativamente con $\% \mathrm{P}$ y $\% \mathrm{H}$ influenciada por las parcelas 9 y 10, la cual estaba correlacionada negativamente con la variable Dr.

De acuerdo con los valores obtenidos del análisis físico (Tabla 2), el comportamiento de las variables en el sistema edáfico se describe de la siguiente manera: la densidad aparente presenta valores altos que responden a la característica textural del suelo, beneficiando aspectos como la porosidad, capacidad de aireación e infiltración. La densidad real presenta valores bajos debido a la característica textural de franco-arenosa que presenta el suelo. El porcentaje de porosidad presenta valores altos a muy altos, indicando una condición porosa ideal para el movimiento de las moléculas de agua y aire en el suelo. El porcentaje de humedad presenta valores medios que se correlaciona con la capacidad porosa del suelo para el almacenamiento de agua, sin embargo, las parcelas 3 y 8 presentan niveles medianamente bajos debido a la evaporación por presentar vegetación herbácea desprovista de árboles y a una desintegración del suelo por procesos de erosión favorecidos por la cercanía al río. El color predominante es el café amarillento, que representa una cantidad relativamente baja de materia orgánica. La textura es franco-arenosa generalizada, la cual mantiene un porcentaje de porosidad y humedad adecuado para el mantenimiento de la fase gaseosa y líquida. La consistencia se presenta en valores nulos como en la parcela 9 que corresponde a una zona de humedad hasta valores relativamente altos como en la parcela 7 donde la compactación del suelo es mayor. La estructura

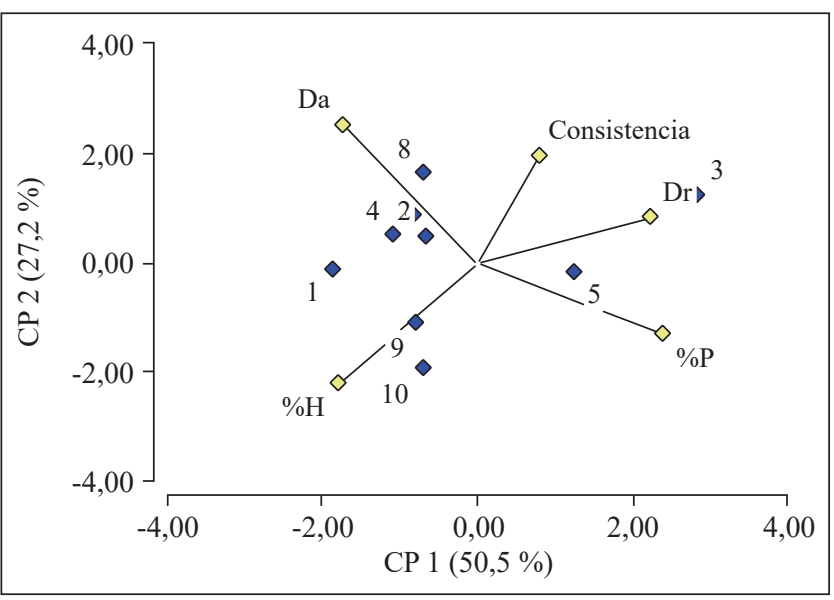

Figura 1. Relaciones entre variables físicas con PCA presentes en los lodos fluviovolcánicos de Armero (Tolima-Colombia). 
Tabla 2. Parámetros físicos evaluados en los lodos fluviovolcánicos de Armero (Tolima-Colombia)

\begin{tabular}{ccccccccl}
\hline Parcela & $\mathbf{D a}$ & $\mathbf{D r}$ & $\mathbf{\%} \mathbf{P}$ & $\mathbf{\%} \mathbf{H}$ & Textura & Estructura & Consistencia & Color \\
\hline 1 & 1,52 & 2,42 & 37,19 & 3,96 & FA & Migajosa & 2,25 & 10 y R-marrón \\
2 & 1,52 & 2,46 & 38,21 & 3,46 & FA & Granular & 2,42 & 10 y R-marrón muy pálido \\
3 & 1,48 & 2,55 & 41,96 & 2,32 & FA & Granular & 3,08 & 10 y R-marrón pálido \\
4 & 1,52 & 2,43 & 37,44 & 3,16 & FA & Migajosa & 1,75 & 2,5 y-marrón amarillento claro \\
5 & 1,47 & 2,50 & 41,20 & 3,34 & FA & Granular & 2,83 & 10 y R- marrón amarillento oscuro \\
6 & 1,40 & 2,47 & 43,30 & 3,10 & FA & Migajosa & 3,00 & 10 y R-marrón pálido \\
7 & 1,53 & 2,41 & 39,04 & 3,34 & FA & Granular & 3,50 & 7,5 y R-gris muy oscuro \\
8 & 1,52 & 2,42 & 37,19 & 2,98 & FA & Granular & 4,00 & 10 y R- marrón amarillento claro \\
9 & 1,49 & 2,44 & 38,93 & 3,40 & FA & Migajosa & 0,00 & 10 y R-marrón muy pálido \\
10 & 1,44 & 2,40 & 40,00 & 4,12 & FA & Granular & 2,20 & 2,5 y- marrón amarillento claro \\
\hline
\end{tabular}

Da: densidad aparente

Dr: densidad real

\%P: porcentaje de porosidad

$\% \mathrm{H}$ : porcentaje de humedad

FA: franco-arenosa

predominante es la granular seguida de la migajosa, lo cual indica un movimiento que los agregados formados permiten un buen movimiento del agua, así como el crecimiento y desarrollo de las raíces de las plantas.

Relación entre variables químicas: los cuatro primeros factores del análisis de componentes principales (PCA) explicaron la variabilidad del $76 \%$, sin embargo, entre los dos primeros se encuentra variabilidad del 50\% (Figura 2). Las parcelas 8, 5 y 2 aportaron un porcentaje de variación del $28 \%$ al factor 1 , mientras que las parcelas $7,4,6$, y 3 aportaron un porcentaje de variación del $22 \%$ al factor 2 .

Las variables $\mathrm{pH}, \mathrm{P}, \mathrm{Mn}, \mathrm{Zn}, \mathrm{Ca}$, saturación de bases, $\mathrm{Mg}, \mathrm{Fe}, \mathrm{Cu}$, relación $\mathrm{Mg} / \mathrm{K}$, relación $(\mathrm{Ca}+\mathrm{Mg}) / \mathrm{K}$, saturación de $\mathrm{Al}$ y concentración de $\mathrm{Al}$ fueron robustas y aportaron información para el análisis.

La variable $\mathrm{pH}$ se correlacionó negativamente con la relación $(\mathrm{Ca}+\mathrm{Mg}) / \mathrm{K}$, relación $\mathrm{Mg} / \mathrm{K}$, saturación de $\mathrm{Al} \mathrm{y}$ concentración de $\mathrm{Al}$. El Na y el $\mathrm{K}$ no presentaron ninguna relación de interacción y se evidenció una correlación positiva entre $\mathrm{P}, \mathrm{Mn}, \mathrm{Ca}, \mathrm{Zn}$, saturación de bases, Fe y $\mathrm{Mg}$.

De acuerdo con los valores obtenidos del análisis químico (Tabla 3), el comportamiento de las variables en el sistema edáfico se describe de la siguiente manera: el $\mathrm{pH}$ se presenta en niveles medianamente ácidos hasta niveles neutros, favoreciendo la movilidad de iones, la precipitación y disolución de minerales, las reacciones redox, el intercambio iónico, la actividad microbiana y la disponibilidad de nutrientes. Sin embargo, puede llegar a ser desfavorable para la solubilidad de la materia orgánica. Los niveles de materia orgánica presentan un contenido relativamente bajo, lo cual determina un aumento en los niveles de $\mathrm{pH}$ y reduce medianamente la disponibilidad de los nutrientes. La capacidad de intercambio catiónico (CIC) presenta unos niveles adecuados para retener y tener a disposición nutrientes procedentes de la meteorización o mineralización de la materia orgánica.

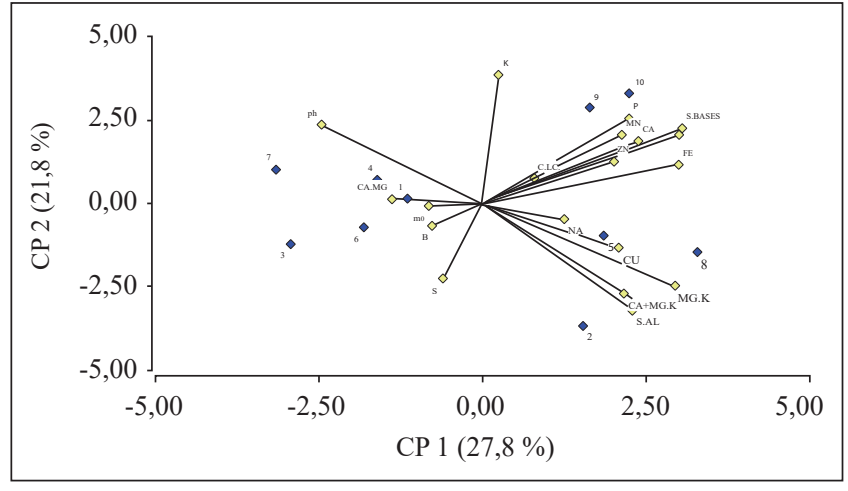

Figura 2. Relaciones entre variables químicas con PCA presentes en los lodos fluviovolcánicos de Armero (Tolima-Colombia).

El P se encuentra en niveles medios, como consecuencia del bajo contenido de materia orgánica que influye en la oferta nutricional de éste elemento; en las parcelas 8, 9 y 10 aunque el contenido de materia orgánica es bajo, el $\mathrm{P}$ es alto debido posiblemente a la C.I.C. que se encuentra en niveles altos.

El Ca en las parcelas 3, 6 y 7 se presentan niveles bajos $\mathrm{y}$ en las otras ya se aprecia un aumento considerable en su concentración. El Mg presenta niveles bajos en las parcelas 2, 3, 4, 5, 6 y 7; en las parcelas 1, 8, 9 y 10 hay un aumento considerable en su concentración.

El Na en las parcelas $1,3,6,7,8$ y 10 presenta niveles bajos y en las parcelas 2, 4, 5 y 9 el valor se encuentra en niveles normales.

El K en las parcelas 2, 3 y 5 presenta niveles muy bajos, en las otras parcelas de igual forma los niveles tienden a hacer bajos, situación que influye en la condición de acidez, al igual que la cantidad de materia orgánica.

El Fe en las parcelas 5, 8, 9 y 10 presenta valores altos que intervienen en las condiciones de acidez y aumentan por el aporte de descomposición mineral. 
Tabla 3. Parámetros químicos evaluados en los lodos fluviovolcánicos de Armero (Tolima-Colombia)

\begin{tabular}{|c|c|c|c|c|c|c|c|c|c|c|}
\hline \multirow[t]{2}{*}{ Parámetros } & \multicolumn{10}{|c|}{ Parcelas } \\
\hline & 1 & 2 & 3 & 4 & 5 & 6 & 7 & 8 & 9 & 10 \\
\hline $\mathrm{pH}$ & 5,70 & 4,00 & 5,80 & 7,20 & 5,00 & 5,50 & 5,50 & 4,00 & 5,60 & 5,40 \\
\hline Materia orgánica & 0,90 & 0,60 & 0,50 & 0,90 & 0,80 & 0,90 & 1,10 & 1,00 & 0,50 & 0,70 \\
\hline Capacidad Intercambio Catiónico & 11,00 & 10,00 & 11,00 & 14,00 & 10,00 & 11,00 & 9,00 & 13,00 & 12,00 & 11,00 \\
\hline Fosforo (P) (ppm) & 27,00 & 34,00 & 52,00 & 54,00 & 55,00 & 53,00 & 44,00 & 81,00 & 93,00 & 83,00 \\
\hline Calcio (Ca) (meq.) & 4,90 & 4,50 & 2,50 & 7,10 & 4,70 & 2,60 & 3,30 & 6,00 & 5,90 & 6,50 \\
\hline Magnesio (Mg) (meq.) & 2,70 & 2,10 & 1,20 & 1,30 & 1,80 & 1,70 & 1,10 & 3,30 & 3,90 & 3,90 \\
\hline Sodio (Na) (meq.) & 0,10 & 0,20 & 0,10 & 0,20 & 0,20 & 0,10 & 0,10 & 0,10 & 0,20 & 0,10 \\
\hline Potasio (K) (meq.) & 0,23 & 0,12 & 0,12 & 0,19 & 0,14 & 0,19 & 0,37 & 0,21 & 0,37 & 0,36 \\
\hline Hierro (Fe) (ppm) & 15,00 & 12,00 & 16,00 & 12,00 & 88,00 & 4,40 & 8,00 & 72,00 & 91,00 & 46,00 \\
\hline Cobre $(\mathrm{Cu})(\mathrm{ppm})$ & 0,80 & 0,80 & 0,20 & 0,30 & 2,60 & 1,80 & 0,30 & 2,00 & 0,20 & 1,20 \\
\hline Zinc $(\mathrm{Zn})(\mathrm{ppm})$ & 1,30 & 1,30 & 0,30 & 2,70 & 3,90 & 3,30 & 1,60 & 2,80 & 2,90 & 2,80 \\
\hline Manganeso (Mn) (ppm) & 9,00 & 9,00 & 5,60 & 7,60 & 32,00 & 9,20 & 6,80 & 12,00 & 12,00 & 56,00 \\
\hline Boro (B) (ppm) & 0,60 & 0,60 & 0,80 & 0,40 & 0,80 & 0,90 & 0,40 & 0,30 & 0,60 & 0,60 \\
\hline Azufre (S) (ppm) & 58,00 & 58,00 & 72,00 & 83,00 & 57,00 & 73,00 & 51,00 & 89,00 & 57,00 & 28,00 \\
\hline Aluminio (Al) (meq.) & 0,00 & 1,60 & 0,00 & 0,00 & 0,60 & 0,20 & 0,20 & 1,60 & 0,00 & 0,20 \\
\hline Saturación de aluminio & 0,00 & 16,00 & 0,00 & 0,00 & 6,00 & 1,80 & 2,20 & 12,30 & 0,00 & 1,80 \\
\hline Saturación de bases & 72,10 & 69,20 & 35,60 & 62,80 & 68,40 & 41,70 & 54,10 & 73,90 & 86,40 & 98,70 \\
\hline Relación $\mathrm{Ca} / \mathrm{Mg}$ & 1,80 & 2,10 & 2,10 & 5,50 & 2,60 & 1,50 & 3,00 & 1,80 & 1,50 & 1,70 \\
\hline Relación $(\mathrm{Ca}+\mathrm{Mg}) / \mathrm{K}$ & 33,00 & 55,00 & 30,80 & 44,20 & 46,40 & 22,60 & 11,90 & 44,30 & 26,50 & 28,90 \\
\hline Relación $\mathrm{Mg} / \mathrm{K}$ & 11,70 & 17,50 & 10,00 & 6,80 & 12,90 & 8,90 & 3,00 & 15,70 & 10,50 & 10,80 \\
\hline
\end{tabular}

El $\mathrm{Cu}$ en las parcelas 1, 2, 3, 4, 7 y 9 presenta niveles bajos, indicando que el $\mathrm{pH}$ al acercarse a la neutralidad reduce su solubilidad en el suelo y los niveles altos que se presentan de Fe también disminuyen su absorción.

El Zn en las parcelas 1, 2, 3 y 7 presentan niveles muy bajos que influyen en la disminución de la acidez y están siendo afectados posiblemente por los niveles de $\mathrm{P}$ que disminuyen su absorción.

El Mn en las parcelas 5 y 10 presentan niveles altos, en las otras parcelas los niveles son bajos lo cual favorece la disminución en la acidez y se ve influido por la cantidad de materia orgánica que aunque es baja va en incremento.

El B en general es bajo e influye en la mediana acidez del suelo y se ve afectado por la textura franco-arenosa.

El S presenta niveles altos en todas las parcelas excepto en la parcela 10 donde existe vegetación arbórea alrededor $\mathrm{y}$ es notable la alteración del terreno debido a siembras y el ganado.

Los niveles variables de $\mathrm{Al}$ y saturación de $\mathrm{Al}$ sólo se registran en las parcelas $2,5,6,7,8$ y 10 , debido a que tienen un $\mathrm{pH}$ más ácido que el resto de las parcelas donde disminuye la movilización y captación del Al.

La saturación de bases $(\mathrm{Ca}+\mathrm{Mg}+\mathrm{Na}+\mathrm{K}) / \mathrm{CIC}) * 100)$ en las parcelas 3, 6 y 7 presenta los niveles más reducidos debido a la baja concentración que tienen de las bases. La relación $\mathrm{Ca} / \mathrm{Mg}$ presenta una calificación de baja a media $\mathrm{y}$ adecuada solo para la parcela 4 , en relación con el $\mathrm{Ca}$ con quien establece una correlación directa. Por otro lado, este valor presenta una calificación muy baja y media solo para la parcela 4 , en relación con el $\mathrm{Mg}$ con quien establece una correlación inversa.

La relación $(\mathrm{Ca}+\mathrm{Mg}) / \mathrm{K}$ presenta un valor medio para las parcelas 1, 2, 3, 4, 5, 6, 7 y 8 y un valor adecuado para las parcela 9 y 10 , se relaciona inversamente con el contenido de $\mathrm{K}$ el cual se encuentra en condiciones bajas. La relación $\mathrm{Mg} / \mathrm{K}$ presenta valores medios en las parcelas excepto en la 7 donde la concentración es baja, teniendo en cuenta, que existe una relación antagónica entre éstos dos elementos y en éste caso la baja presencia de $\mathrm{K}$ aumenta los niveles de magnesio.

Variación de las condiciones edáficas en el tiempo: de acuerdo con los datos de la tabla 4 en el primer estudio de sucesión vegetal y edafológica se reportaron unos niveles altos de S (1463 ppm) y de Fe (278,8 ppm), un porcentaje de acidez alto $(3,3)$ y un contenido de materia orgánica bajo; mientras que para el segundo estudio se presentó una reducción significativa de $\mathrm{S}$ (413 ppm) y el Fe (136,6 ppm) en un $70 \%$ y $50 \%$ respectivamente, el pH mostró una disminución en su acidez $(4,34)$ y el incremento de materia orgánica fue alto.

En el tercer estudio se evidenció una reducción considerable en los niveles de S (182.47 ppm) y de Fe $(20,88$ $\mathrm{ppm})$, un aumento en el $\mathrm{pH}(5,12)$ y una reducción muy baja en los niveles de materia orgánica. Para el cuarto estudio se reportó una continua reducción en el $\mathrm{S}(62,6 \mathrm{ppm})$ y un incremento en el contenido de Fe (36,44 ppm); el valor del 
Tabla 4. Parámetros químicos comparables en los cuatro estudios de sucesión vegetal de los lodos fluviovolcánicos de Armero (TolimaColombia)

\begin{tabular}{ccccccccccc}
\hline Estudio & $\mathbf{p H}$ & Materia orgánica & $\mathbf{S}(\mathbf{p p m})$ & $\mathbf{P}(\mathbf{p p m})$ & $\mathbf{K}(\mathbf{m e q})$. & $\mathbf{C a}(\mathbf{m e q})$. & $\mathbf{M g}(\mathbf{m e q})$. & $\mathbf{F e}(\mathbf{p p m})$ & $\mathbf{Z n}(\mathbf{p p m})$ & $\mathbf{M n}(\mathbf{p p m})$ \\
\hline 1 & 3,30 & 0,38 & 1463,00 & 104,70 & 0,03 & 5,10 & 3,08 & 278,80 & 17,80 & 100,00 \\
2 & 4,34 & 1,44 & 413,00 & 107,20 & 0,178 & 7,24 & 3,42 & 136,60 & 3,72 & 23,76 \\
3 & 5,12 & 1,11 & 182,47 & 56,30 & 0,35 & 6,99 & 1,53 & 20,88 & 5,93 & 17,52 \\
4 & 5,37 & 0,79 & 62,60 & 57,60 & 0,23 & 4,80 & 2,30 & 36,44 & 2,29 & 15,92 \\
\hline
\end{tabular}

pH se encontró con una baja acidez y los contenidos de materia orgánica presentaron una reducción. En los cuatro estudios realizados se reportó una textura franco-arenosa.

Análisis suelo-vegetación: en los cuatro estudios de sucesión vegetal realizados en la zona de Armero afectada por los lodos fluviovolcánicos se registraron 565 especies distribuidas en 72 familias, de las cuales sobresalen las familias Leguminosae, Poaceae, Asteraceae, Malvaceae, Euphorbiaceae y Cyperaceae. Es evidente que el cambio en las diferentes concentraciones de los parámetros físicos y químicos ha sido proporcional a la variación en la vegetación, encontrándose el predominio de hierbas en un $45 \%$, y árboles en un $20 \%$.

\section{Discusión}

Las condiciones edáficas que se presentaron después de la deposición de los lodos favorecieron en un primer momento la aparición de 294 especies que colonizaron a través de la formación de montículos, siendo las especies más notables Sarcostemma clausum, Melinis repens (Rhynchelitrum repens) y Cyperus ligularis (Mariscus ligularis). En el segundo estudio la riqueza florística se aumentó en 38 especies, conformando cuatro asociaciones básicas que fueron Rhynchelitretum, Indigoferetum, Ficetum y Panicetum; la segunda asociación dominada por especies del grupo de las leguminosas fue determinante en el proceso de cambio de las condiciones edáficas al contribuir a la fertilización de los lodos por la acción de los nódulos con bacterias nitrificantes presentes en las raíces. Para el tercer estudio solo las asociaciones vegetales Panicetum y Ficetum fueron notables y se notó un amplio cubrimiento de las leguminosas en todos los sectores; el número de especies disminuyó considerablemente y para el último estudio se registraron 195 especies, con dominio del hábito de crecimiento arbóreo y arbustivo. Es evidente la acción de las leguminosas como grupo funcional clave en el mejoramiento de la calidad del suelo, teniendo en cuenta, los servicios agroecológicos que proporcionan en cuanto al aumento de la vegetación, incremento de biomasa e influencia positiva en la abundancia y diversidad microbiana (Duchene, et al., 2017).

Por lo anterior, se puede inferir que el asentamiento de nuevas especies vegetales sobre los lodos está relacionado con los cambios en las condiciones edáficas, y de acuerdo con los planteado por Arbelo, et al., (2002), esto influye para que las formaciones actuales de vegetación boscosa se establezcan en condiciones favorables para su desarrollo, reflejado en mayor contenido de materia orgánica y la disminución de los niveles de $\mathrm{Fe}, \mathrm{S}$ y $\mathrm{Al}$, los cuales en altas concentraciones generan toxicidad y acidez del suelo, así como la inhibición de actividades enzimáticas mediadas por microbios en los ciclos de nutrientes (Kunito, 2016).

Actualmente existe una similitud en la vegetación entre el primer y último estudio de sucesión debido a la fuertes actividades de intervención a la que se ha visto sometida el área de Armero entre las que se encuentra la agricultura intensiva, el monocultivo de especies promisorias y quemas, las cuales modifican la estructura física del suelo y altera la concentración de los nutrientes disponibles haciendo que el proceso de sucesión se reinicie con el establecimiento de especies pioneras, esto es similar a lo planteado por Evans (2006) quien indica que la sucesión no es unidireccional sino que por el contrario es un proceso dinámico que puede presentar un retroceso. De igual forma, la vegetación continúa en un cambio progresivo en su estructura y composición y a pesar de que el cambio de la comunidad vegetal esté fuertemente relacionado con la variación de las condiciones del suelo, estos procesos se dan a largo plazo (Rodríguez, Arbelo, Guerra, Mora, 2001).

En cuanto a las variables físicas y químicas del suelo y su influencia en la vegetación a través de los años, Esquivel, Ramírez (1994) reportaron una íntima relación entre el proceso de sucesión vegetal y la evolución de las características del suelo. Según Gómez (1988) la textura franco arenosa presenta una adhesividad media, una infiltración buena, una retención de humedad regular y una aireación buena conformándose en éste suelo una textura que tiende al equilibrio y dispone de unas condiciones efectivas para el desarrollo de las funciones fisiológicas de las plantas (Fajardo, 2005). Sin embargo, en suelos de origen volcánico es notable la combinación textural que proporciona distribuciones de minerales frecuentemente homogéneas, con la acción de elementos como el $\mathrm{Mg}$ y $\mathrm{Ca}$ que actúan en su movilidad y estabilidad (Cuadros, 2016).

Los elevados contenidos de Fe y $\mathrm{S}$ que se reportaron en el primer estudio son consecuentes por la alta carga de estos elementos que fueron depositados por los lodos volcánicos. A medida que se inició el crecimiento de la vegetación mediante una sucesión primaria, los niveles de éstos elementos se redujeron considerablemente por la incorporación realizada a las plantas, especialmente en las forrajeras (Malavolta, 1985). 
La acidez del suelo al estar relacionada con las propiedades físicas y químicas ha sido fundamental para el establecimiento de la vegetación actual, el cual ha presentado una disminución progresiva de la acidificación, favoreciendo la disponibilidad y asimilación de los nutrimentos por las plantas (Marín, Lora, 1979).

El contenido de la materia orgánica ha sido favorecido por la temperatura (Lora, 1971), también la textura francoarenosa ha permitido el balance entre los procesos de aporte al suelo; de igual forma las condiciones cercanas de neutralidad que se presentan en el suelo ha favorecido la descomposición de la materia orgánica dentro de los rangos ideales para la obtención de productos aprovechables por las plantas (Raheja, 1966). Según Taboada (2016), el grado de meteorización, el horizonte del suelo y las zonas bioclimáticas están relacionadas con la cantidad de materia orgánica ofertada por el suelo, lo cual sugiere que la ubicación geográfica y altitudinal de Armero ha contribuido con la descomposición y aprovechamiento de los elementos químicos presentes en el medio edáfico.

Las concentraciones de $\mathrm{K}$ en los últimos 25 años se han elevado lo cual ha significado un beneficio para el establecimiento de la vegetación, teniendo en cuenta que, es un macronutriente absorbido por las plantas en grandes cantidades, siendo superado sólo por el nitrógeno y, a veces por el Ca (Sanzano, 2001). Los niveles de P han mantenido un comportamiento de disminución a través de los años, posiblemente por el porcentaje de materia orgánica pero, sin embargo, sigue con un efecto positivo que da continuidad al proceso de sucesión (Ramírez, 1994). Estas variaciones de las condiciones químicas se sustentan en el tiempo por la disponibilidad y reserva de nutrientes que ha generado la disposición de los lodos fluviovolcánicos, a través de largos procesos de intemperización que generan oferta de nutrientes suficientes para satisfacer las necesidades ecológicas de restauración y producción (Anda, 2015).

\section{Conclusiones}

Las características físicas y químicas del suelo se modificaron drásticamente durante los 30 años posteriores a la erupción volcánica y actualmente las variables presentan niveles aptos para el desarrollo de la vegetación. Han pasado de un sistema de acidez, salinidad y sobresaturación de elementos al establecimiento de un ecosistema edáfico propicio para el desarrollo de especies típicas de la formación vegetal de bosque seco tropical.

Las variables físicas del suelo han establecido condiciones para que la vegetación se desarrolle, a través de niveles óptimos de humedad, porosidad y una estructura que facilita el desplazamiento y la toma de los nutrientes por parte de las raíces.

El pH ha incrementado, pero la mayor parte de los lodos se mantiene en un nivel moderado de acidez que no afecta drásticamente la vegetación, pero, disminuye la concentración de materia orgánica y de elementos como el
P y el K, sin embargo, otros elementos como el Fe presenta niveles adecuados para su participación en el mantenimiento y la demanda fisiológica de la planta.

Las condiciones edáficas han estado directamente relacionadas con el establecimiento de la vegetación sucesional en un proceso ecológico alterno a las perturbaciones naturales y humanas, lo cual se expresa en la evidente sucesión a tal punto que especies que fueron dominantes en los primeros años del proceso, actualmente son escasas como sucede con Melinis repens (Poaceae), Sarcostemma clausum (Apocynaceae), Cyperus ligularis (Cyperaceae) $e$ Indigofera hirsuta (Leguminosae), entre otras.

\section{Agradecimientos}

Los autores expresan sus agradecimientos a la Oficina de Investigaciones y Desarrollo Científico de la Universidad del Tolima por la financiación del proyecto.

\section{Conflicto de intereses}

Los autores declaran no tener conflicto de intereses.

\section{Referencias}

Alfaro, E., Alvarado, A. \& Chaverri, A. (2001). Cambios edáficos asociados a tres etapas sucesionales del bosque tropical seco en Guanacaste. Agronomía Costarricense. 25: 7-20.

Anda, M., Suryani, E. \& Djadja, H. (2015). Strategy to reduce fertilicer application in volcanic paddy soils: Nutrient reserves approach from parent materials. Soil and Tillage Research. 150: 10-20.

Arbelo, C., Guerra, J., Mora, J. \& Laguna L. (2002). Calidad del suelo y sucesión vegetal en andosoles forestales de las islas canarias. Edafología. 9: 31-38.

Castillo, A. \& Salinas, N. (1992). Análisis de los lodos de Armero seis años después de la avalancha en la parte alta-mediabaja y su vegetación alrededor del Rio Lagunilla. Ibagué: Universidad del Tolima.

Cantillo, E., Lozada, A. \& Pinzón, J. (2009). Caracterización sucesional para la restauración de la reseva forestal Cárpatos, Guasca, Cundinamarca. Revista Colombia Forestal. 12: 103-118.

Cuestas, P. (2005). El análisis de suelos: Toma de muestras y recomendaciones de fertilización para la producción ganadera. En Corpoica-MADR (ed). Producción y utilización de recursos forrajeros en sistemas de producción bovina de las regiones caribe y valles interandinos. (pp. 1-10). Bogotá.

Cuadros, J., Díaz, J., Sanchez, A., García, A. \& Yepes, J. (2016). Chemical and textural controls on the formation of sepiolite, palygorskite and dolomite in volcanic soils. Geoderma. 271: 99-114.

Cháves, H. \& Guevara, J. (1987). Delimitación de los suelos (protosuelos). En IGAC (ed). Proyecto de restauración forestal y corrección de torrentes en las zonas afectadas por la erupción del Volcán Nevado del Ruíz. Bogotá.

Duchene, O., Vian, F. \& Celette, F. (2017). Intercropping with legume for agroecological cropping systems: Complementarity and facilitation procesess and the importance of soil microorganisms. A review. Agriculture, Ecosystems \& Environment. 240: 148-161. 
Esquivel, H. \& Leguizamón, G. (1989). Estudio florístico de la región cubierta por los lodos fluviovolcánicos en la zona, como consecuencia de la erupción del Vocán Nevado del Ruiz en 1985. Ibagué: Universidad del Tolima.

Esquivel, H. \& Ramirez, L. (1994). Estudio sucesional de los lodos fluviovolcánicos del municipio de Armero. Ibagué: Universidad del Tolima.

Esquivel, H., Frye, A. \& Ramírez, L. (1999). Estado de la sucesión vegetal y edafológica de los lodos de Armero 15 años después de la erupción del Volcán Arenas del Nevado del Ruiz. Ibagué: Universidad del Tolima.

Evans, M. (2006). Caracterización de la vegetación natural de sucesión primaria en el Parque Nacional Volcán Pacaya y Laguna de Calderas. Guatemala: Centro Agronómico Tropical de Investigación y Enseñanza (CATIE).

Fajardo, N. (2005). Uso y manejo de suelos. Ibagué: Universidad del Tolima.

FAO. (1990). Guidelines for soil profile description. Holanda: ROME/ISRIC, Wageningen.

Frye, A. (1986). Caracterización físico-química y diagnóstico edafológico de los aluviones volcánicos de Armero. Ibagué: Universidad del Tolima.

Gomez, A. (1988). La zona andina colombiana. Erosión y conservación de suelos. Chinchiná, Caldas: CENICAFÉ.

Holdridge, L. (1979). Ecología basada en zonas de vida. San José, Costa Rica: Centro Agronómico Tropical de Investigación y Enseñanza (CATIE).

ICA. (1992). Fertilización en diversos cultivos. Bogotá: PRODUMEDIOS.

Kunito, T., Isomura, I., Sumi, H., Park, H., Toda, H., Otsuka, S., Nagaoka, K., Saeki, K. \& Senoo, K. (2016). Aluminum and acidity supress microbial activity and biomass in acidic forest soils. Soil Biology and Biochemistry. 97: 23-30.
Loaiza, J. (2011). El recurso suelo. Suelos ecuatoriales. 41: 6-18.

Lora, S. (1971). Materia orgánica y nitrógeno en el suelo. Interpretación de análisis de suelos y recomendaciones de fertilizantes. Bogotá: Instituto Colombiano Agropecuario (ICA).

Malagón, D. (1989). El flujo de los lodos en la región de Armero (Tolima-Colombia). Caracterización y Manejo inicial. En Resurgir (ed). Aspectos médicos de la catástrofe volcánica del Nevado del Ruíz. (pp. 49-52). Bogotá. Instituto Geográfico Agustín Codazzi (IGAC).

Malavolta, E. (1985). Desordens nutricionais no cerrado. Piracicaba, Brasil: POTAFOS.

Marin, G. \& Lora, R. (1979). Acidez y encalamiento de suelos. Bogotá: Instituto Colombiano Agropecuario (ICA).

Raheja, P. (1966). Organic matter and carbón-nitrogen relationships. Soil productivity and crop growth. New York, Estados Unidos: P. HOUSE.

Rodriguez, A., Arbelo, C., Guerra, J. \& Mora, J. (2001). Influences of changes in use on the properties of andosols and andic soils. Volcanic soils: Properties, processes and land use internat. Azores, Portugal.

Sanzano, A. (2001). El Potasio del suelo. Argentina: Cátedra de Edafología, Facultad de Agronomía y Zootecnia, UNT.

Taboada, T., Rodríguez, L., Ferro, C., Stoops, G. \& Martínez, A. (2016). Chemical weathering in the volcanic soils of Isla Santa Cruz (Galápagos Islands, Ecuador). Geoderma. 261: 160-168.

Torres, A., Adarve, J., Cárdenas, M., Vargas, J., Londoño, V., Rivera, K., Home, J., Duque, O. \& González, A. (2012). Dinámica sucesional de un fragmento de bosque seco tropical del Valle del Cauca, Colombia. Biota Colombiana. 13: $66-88$. 